W1E-(12)-2

\title{
MYOCARDIAL DIAGNOSIS BY USING POLARIZATION-SENSITIVE OPTICAL COHERENCE TOMOGRAPHY
}

\author{
Chia-Wei Sun, Yih-Ming Wang, I-Jen Hsu, Chih-Wei Lu, Yean-Woei Kiang and C. C. Yang \\ Graduate Institute of Electro-Optical Engineering and Department of Electrical Engineering, \\ National Taiwan University, Taipei, Taiwan, R.O.C. \\ (phone) 886-2-23657624 (fax) 886-2-23652637 (E-mail) ccy@cc.ee.ntu.edu.tw \\ Long-Sheng Lu \\ Graduate Institute of Pharmacology, National Taiwan University, Taipei, Taiwan, R.O.C.
}

Abstract-- A polarization-sensitive optical coherence tomography system based on a 12-fsec Ti:sapphire laser as the broadband source was used to ex vivo diagnosing diet-induced hypercholesterolemia myocardium of New Zealand white rabbits.

Most heart diseases are associated with structural changes, either in composition or in the arrangement of muscle fibers within the myocardium. To determine the optimal treatment strategy with such injuries, it is important for cardiovascular specialists to know the distributions as well as the sizes of lesions. Therefore, polarization-sensitive optical coherence tomography (PSOCT) can be of great help for myocardium tissue characterization and heart disease diagnosis. Usually, Super-luminescent diodes were used for PSOCT operation and hence typically 10-15 $\mu \mathrm{m}$. longitudinal resolution was achieved. To improve the resolution, a mode-locked Ti:sapphire laser with spectral width up to $80-100 \mathrm{~nm}$ was chosen as the broadband light source in our PSOCT system. In the PSOCT system, a Verdi-laser-pumped mode-locked Ti:sapphire laser provided $100 \mathrm{fsec}, 76 \mathrm{MHz}$ pulses with the central wavelength at $800 \mathrm{~nm}$. The interfered signals were processed with two photo-detectors and band-pass filters to extract the images of the sub-surface morphology with different polarization components. The full-widths-at-half-maximum of the two interference fringe pattems were about $10 \mu \mathrm{m}$, corresponding to the longitudinal resolution of the PSOCT system in air. This resolution cell size is lager than the theoretical value. This is so because of the dispersion mismatch between the reference and sample arms. In our experiments, the New Zealand white rabbits were fed with either standard or $0.5 \%$ cholesterol-enriched chow for 12 weeks. To harvest myocardial tissues, lethal dose of sodium pentobarbital was administered. Animals were retrogradely perfused with heparinized PBS (10 units $/ \mathrm{ml}$ ) for 15 minutes followed by $2 \%$ paraformaldehyde ( $\mathrm{pH} 7.4$ ) for another 15 minutes. Then, the hearts were removed quickly and stored in $10 \%$ formaldehyde till the optical experiments. $4 \mathrm{~mm}$ slabs of mid-ventricular cross-section were used for PSOCT imaging. Figure 1 shows the channel 1 image of normal myocardium with horizontal polarization input. The images from the two channels furnish the birefringence information of the myocardium tissue. The difference between the two images illustrates the different fibrous arrangements in the transverse direction. The PSOCT images of diet-induced hypercholesterolemia myocardium are shown in Fig. 2 (Channel 2 with vertical polarization input). Again, the two images of the two channels in this case reveal the birefringent structures. Microscopically, hearts from hypercholesterolemic rabbits were examined to find increased density of small-caliber vessels and perivascular lipid-laden macrophages. Atherosclerotic lesions were found in small perforating branches but not in epicardial coronary arteries. Cavity structures in Fig. 2 can be clearly seen. The comparison between Figs. 1 and 2 shows the significant differences between normal and abnormal myocardial tissues.

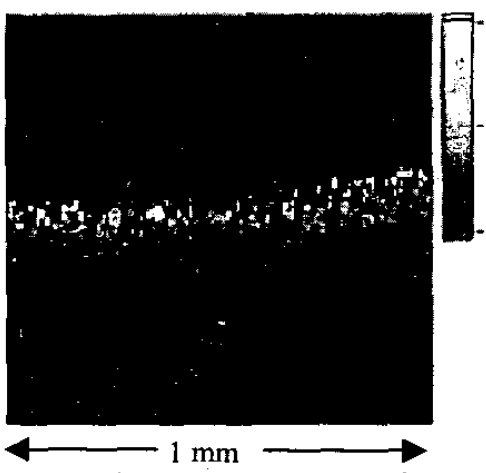

Fig. 1 OCT myocardium images of a normal New Zealand white rabbit from channel 1 with horizontal polarization input.

0-7803-7766-4/03/\$17.00 @2003 IEEE

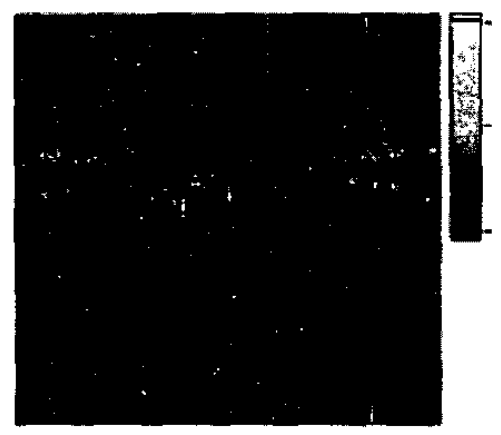

Fig. 2 OCT myocardium images of a diet-induced hypercholesterolemia New Zealand white rabbit from Channel 2 with vertical polarization input. 